This is the final peer-reviewed accepted manuscript of:

F. Morandi, M. Miniaci, A. Marzani, L. Barbaresi, M. Garai, Standardised acoustic characterisation of sonic crystals noise barriers: Sound insulation and reflection properties, Applied Acoustics, Volume 114, 2016, Pages 294-306, ISSN 0003-682X

The final published version is available online at:

https://doi.org/10.1016/j.apacoust.2016.07.028

Rights / License:

The terms and conditions for the reuse of this version of the manuscript are specified in the publishing policy. For all terms of use and more information see the publisher's website.

This item was downloaded from IRIS Università di Bologna (https://cris.unibo.it/)

When citing, please refer to the published version. 


\title{
Standardised acoustic characterisation of sonic crystals noise barriers: sound insulation and reflection properties
}

\author{
F. Morandi ${ }^{\mathrm{a}}$, M. Miniaci ${ }^{\mathrm{b}}$, A. Marzani ${ }^{\mathrm{c}}$, L. Barbaresi $^{\mathrm{a}}$, M. Garai $^{\mathrm{a}}$ \\ ${ }^{a}$ DIN, University of Bologna, viale Risorgimento 2, 40136 Bologna, Italy \\ ${ }^{b}$ Laboratoire Ondes et Milieux Complexes, UMR CNRS 6294, University of Le Havre, 75 rue Bellot, 76600 Le Havre, France \\ ${ }^{c}$ DICAM, University of Bologna, viale Risorgimento 2, 40136 Bologna, Italy
}

\begin{abstract}
This work presents sound insulation and sound reflection measurements conducted over sonic crystal noise barriers according to the European standards EN 1793-2, EN 1793-5 and EN 1793-6. In most of the reference literature, sound insulation and reflection properties of sonic crystals are measured or a diffuse sound field or in a direct sound field including the top and side edge diffraction effects together with the transmitted (or reflected) components. The aim of this work is to perform free-field measurements over a real-sized sample in order to window out all diffraction components and to verify the points of strength and weakness of the application of standardised measurements to sonic crystals. Diffuse field measurements in laboratory are also done for comparison purposes. Since the target frequency range for traffic noise spectrum is centred at around $1000 \mathrm{~Hz}$, a finite element based parametric investigation is performed to design unit cells capable of generating band gaps in the one-third octave bands ranging from $800 \mathrm{~Hz}$ to $1250 \mathrm{~Hz}$. Then, $3 \times 3 \mathrm{~m}$ sonic crystal noise barriers are installed in the Laboratory of the University of Bologna and sound insulation and sound reflection measurements are performed according to the mentioned active standards for normal incidence. Sound insulation is measured for diffuse incidence too. The two methods give different results. The method more directly comparable to calculations is the free-field one. However, if on the one hand the application of a time window allows to compute the transmitted or reflected component only, on the other hand the time window itself limits the maximum width of the sample for which all reflections of the $n-t h$ order having a significant spectral content are included, and thus results critical in the analysis of this kind of noise barriers. Nevertheless, the standardised measurements allow a direct comparison between the performance of sonic crystals and common noise barriers.
\end{abstract}

Keywords: Sonic Crystals, Noise Barriers, Sound Insulation Index, Sound Reflection Index, EN 1793-6, EN 1793-5

\section{Introduction}

In the last two decades, many works have been devoted to the investigation of sound waves propagating through a periodic distribution of solid elements, generally known as sonic crystals. The ability of sonic crystals to act as stop-band filters in the audible frequency range, i.e. to attenuate waves over frequency bands called band gaps, is the most attractive and studied property of such materials. In addition, sonic crystal barriers leave air and light to flow trough, particularly suitable in urban contexts. Since pioneering works showed the existence of band gaps in sonic crystals [1-3], there have been efforts to search wider and frequency-tunable band gaps by varying the mechanical properties of the solid

Email address: massimo.garai@unibo.it (M. Garai) inclusions as well as their spatial arrangement [4-10]. In this context, experimental results showing that simple lightweight sonic crystals are able to reduce sound transmission up to $25 \mathrm{~dB}[2,11]$ have led to a growing interest in exploiting the potential of sonic crystals as acoustic filters and noise barriers. To enhance sound insulation properties of sonic crystals, researchers have recently focused on systems in which both Bragg scattering and local resonant phenomena apply [12-16], on the application of absorbing materials to the scatterers [17-20] as well as on the introduction of lattice defects $[21,22]$. These investigations showed that periodic arrays of scatterers composed of a low number of elements are capable of achieving sound attenuation values large enough to compete with other acoustic screening techniques. On the other hand, the "intrinsic" acoustic characterisation of a noise barrier in itself as a "prod- 
uct" requires assessment of the transmitted and reflected sound properties [23]. In order to measure the effective screening effect of the barrier, diffraction effects and all unwanted reflections should be cut off from the measurements. This is possible, even in a non-anechoic environment, using transient sound signals and a suitable windowing technique, which is currently standardised in EN 1793-5 and EN 1793-6 [25, 26]. In most of the previous literature on sonic crystals, the existence of band gaps is experimentally verified on finite size samples measured in anechoic chambers, see for instance Refs. [2, 15, 20]. However, the relative source-samplemicrophone position does not allow to isolate the transmitted component only; the sound diffracted over the top edge of the sample and around its sides mixes up with the sound field transmitted through the sample. Thus, this kind of measurement yields a rough value of insertion loss. Two significant exceptions to this measurement method are Refs. [15, 11]. In Ref. [15] the impulse responses are windowed in order to minimise the reverberation effects, which were evident in the freefield measurements. A window of $6 \mathrm{~ms}$ is used, but since the rods were $1 \mathrm{~m}$ long, diffraction effects from the top of the barrier were taken into consideration. In Ref. [11] measurements were conducted over a $1.1 \times 7.2$ $\mathrm{m}$ sample. The width of the sample was kept large in order to minimise the edge diffraction and measurements were performed in open air. The work demonstrated that the sound attenuation values provided by sonic crystals are large enough to compete with standard noise barriers; acoustic measurements were not performed under the specifications of the direct field method [25, 26] though. In more recent years, Castineira-Ibànez et al. [8] characterised acoustic barriers based on fractal geometries to maximise the Bragg scattering and multiphenomena scatterers with several noise control mechanisms, as resonances or absorption, by acoustic standardisation tests according to EN 1793-1, EN 1793-2 and EN 1793-3, i.e. under diffuse field conditions.

In this scenario, the present work aims at designing sonic crystal noise barriers based on uniform scatterers and to determine experimentally their acoustic performance according to the EN 1793-2, EN 1793-5 and EN 1793-6 standards [24-26]. These references were chosen because they describe a method which allows one to perform laboratory measurements returning results which do not differ significantly from in situ measurements [27]. As such, the analysis of standard sound insulation index $(S I)$ and reflection index $(R I)$ values for sonic crystals allows a direct comparison between these barriers and the common ones, comparison which to the authors' knowledge has not been performed yet. The

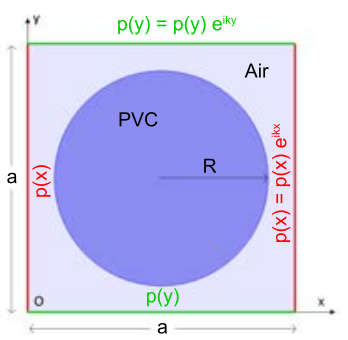

(a)

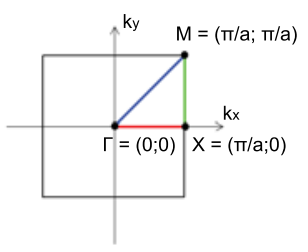

(c)

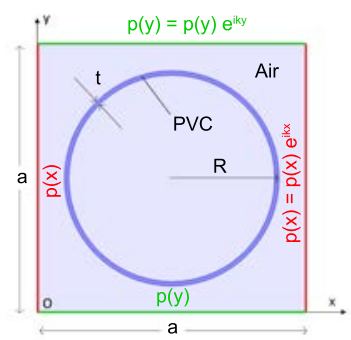

(b)
Figure 1: (a) Air-PVC rod unit cell; (b) air-PVC hollow cylinder unit cell analysed in the present work and (c) first Brillouin zone. Lattice constant is $0.20 \mathrm{~m}$, outer radius is $0.08 \mathrm{~m}$ and thickness is $3.2 \mathrm{~mm}$.

study is organised as follows. First, single unit cells are studied by means of finite element (FE) Bloch-type analyses in order to investigate the band structure of the unit cells able to generate band gaps in the one-third octave bands from $800 \mathrm{~Hz}$ to $1250 \mathrm{~Hz}$. Next, full-scale samples made of PVC cylinders are arranged varying the number of rows and measurements are carried out following the European standards EN 1793-2, EN 17935 and EN 1793-6 [24-26]. Time harmonic FE analyses of the full scale samples are performed to support the experimental interpretation. In particular, the experimental results are compared with the numerical ones in order to prove the existence of band gaps in the targeted frequency range and to determine the standardised acoustic performance of the designed barriers. Finally, a comparison between sound insulation and reflection indices measured for a 3-rows sonic crystal and standard noise barriers is presented; strengths and weaknesses of sonic crystal barriers with reference to ordinary noise barriers are highlighted and related to the measurement system.

\section{Extraction of band structures}

Numerical analyses have been performed to design a sonic crystal exhibiting a complete band gap in the onethird octave bands from $800 \mathrm{~Hz}$ to $1250 \mathrm{~Hz}$, i.e. the frequency range in which tyre/road noise spectrum shows 
Table 1: Properties of the materials used in the FE analyses: density $\rho$, longitudinal wave speed $c_{L}$ and shear wave speed $c_{S}[30,31]$.

\begin{tabular}{cccc}
\hline Material & $\rho\left[\mathrm{kgm}^{-3}\right]$ & $c_{L}\left[\mathrm{~ms}^{-1}\right]$ & $c_{S}\left[\mathrm{~ms}^{-1}\right]$ \\
\hline Air & 1.25 & 343 & - \\
PVC & 1400 & 2142 & 874 \\
\hline
\end{tabular}

a prominent peak [28]. The lattice constant is set in accordance to the Bragg scattering theory; looking for a band gap in the one-third octave band with centre frequency at approximately $1000 \mathrm{~Hz}$, the resulting lattice constant is $0.17 \mathrm{~m}$, given $c_{\text {air }}=343 \mathrm{~m} / \mathrm{s}$ the speed of sound in air at $20^{\circ} \mathrm{C}$. Herein, a lattice constant of 0.20 $\mathrm{m}$ is chosen for the unit cell, expecting the first Bragg frequency at around $860 \mathrm{~Hz}$. Two different inclusions have been considered: (i) a PVC rod of radius $0.08 \mathrm{~m}$ placed at the centre of the unit cell and (ii) a hollow PVC cylinder with outer radius $0.08 \mathrm{~m}$ and thickness of 3.2 mm.

Unit cells are presented in Fig. 1 (a) and (b) along with proper periodic boundary conditions in terms of pressure distribution, while the properties of the materials used in the calculations are listed in Tab. 1. For both cases, band structures are computed along the three high symmetry directions of the first irreducible Brillouin zone $\Gamma X, X M$ and $M \Gamma$ using the FE method and exploiting the Bloch-Floquet theorem with the aid of the commercial software Comsol MultiPhysics $4.3^{\circledR}$ [29]. The unit cell domains are modeled under 2D plane strain assumption exploiting the "Acoustic-Solid interaction" module and meshed by means of 3-node triangular elements of maximum edge length $L_{F E}=0.01 \mathrm{~m}$ to provide accurate eigensolutions up to the maximum frequency of $2500 \mathrm{~Hz}$. Case (ii) required a mesh refinement up to $L_{F E \text { min }}=3.2 \cdot 10^{-3} \mathrm{~m}$ for the inclusion. The resulting Bloch-type eigenvalue problems are solved using the PARDISO algorithm for the $\Gamma X, X M$ and $M \Gamma$ paths. A detailed description of the procedure to extract the band structures can be found in Refs. [30, 31].

Fig. 2 presents the band structures in terms of reduced wavevector $\mathbf{k}^{*}=\left[k_{x} a / \pi ; k_{y} a / \pi\right]$, where $k_{x}$ and $k_{y}$ are the wavevectors in the $x$ and $y$ directions, respectively. At least one complete band gap (dark grey rectangle) as well as partial band gaps (light grey rectangles) exist in the $0-2500 \mathrm{~Hz}$ frequency range for each unit cell. In particular, a complete band gap extends from $858 \mathrm{~Hz}$ to $1107 \mathrm{~Hz}$ for case (i) and from $867 \mathrm{~Hz}$ to $1117 \mathrm{~Hz}$ for case (ii). In the latter case, an additional lower complete band gap between 145 and $202 \mathrm{~Hz}$ is generated. Fig. 2 suggests that, due to the high acoustic

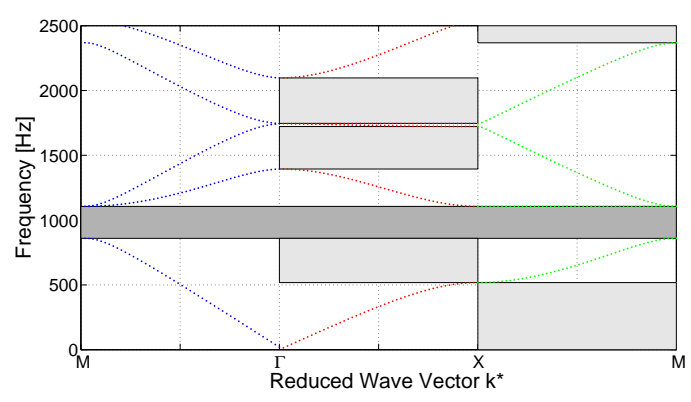

(a)

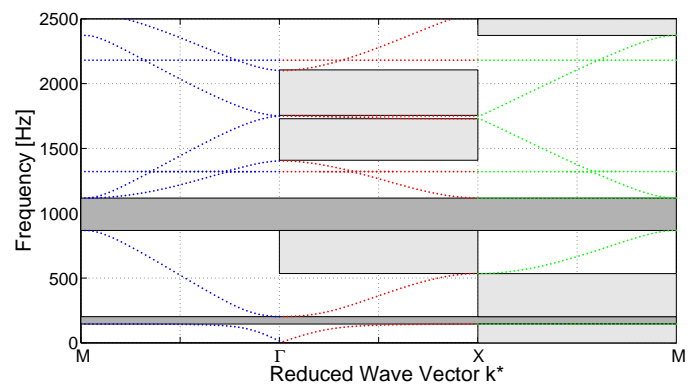

(b)

Figure 2: Band structures for (a) air-PVC bar unit cell and (b) air-PVC hollow cylinder unit cell in the first irreducible Brillouin zone.

mismatch between air and PVC, the complete band gap centred at around $1000 \mathrm{~Hz}$ mainly depends on the outer shape of the inclusion rather than on the geometrical differences of the considered inclusions (filled or hollow), as already pointed out in Ref. [32]. Thus, in the following while barriers of hollow cylindrical pipes will be tested experimentally, numerical FE harmonic simulations will consider filled pipes to alleviate the computational needs. Finally, it is worth noting that some waves coalesce at the high symmetry point $M$ outside the band gap boundaries, as it occurs between $2^{\text {nd }}$ and $3^{\text {rd }}$ curves for the case (i) or between $3^{\text {rd }}$ and $4^{\text {th }}$ curves for the case (ii).

\section{Measurement setup}

The intrinsic properties of a noise barrier can be characterised according to standardised criteria, which have the advantage to allow a straightforward comparison between sonic crystals and other acoustic screens. Real-sized sonic crystal noise barriers have been set in the Acoustic Laboratory of the University of Bologna. Sound insulation measurements have been conducted under direct sound field, following the EN 1793-6 [26] 


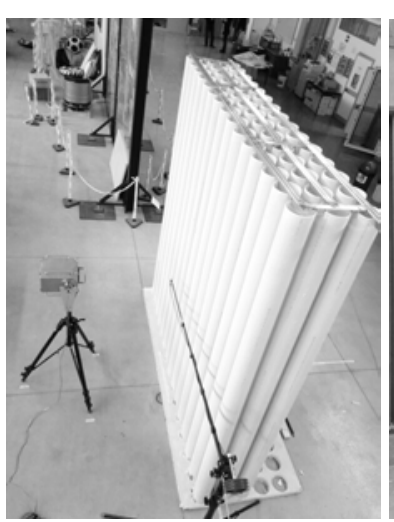

(a) $S I$ setup (b) $R$ setup

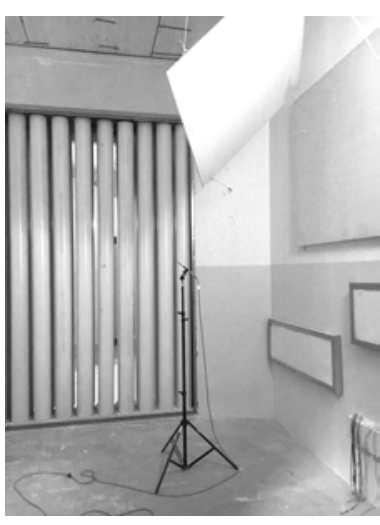

Figure 3: Sound Insulation Index (a) and Sound Reduction Index (b) setups for a 3-rows sonic crystal barrier.

standard and under diffuse sound field conditions, following the EN 1793-2 [24] standard for the laboratory characterisation of barriers. Reflection properties have been characterised according to the standard EN 1793-5 [25] under direct sound field conditions.

As far as it concerns measurements under direct field conditions, the sonic crystal was arranged in the main hall of the laboratory, an industrial hall with a volume of approximately $5000 \mathrm{~m}^{3}$ (Fig. 3 a). The sample is made of hollow PVC cylinders arranged in a square lattice as shown in Fig. 4. The cylinders stand vertically with a length of $3 \mathrm{~m}$ and have with an outer radius of $0.08 \mathrm{~m}$ and thickness $3.2 \mathrm{~mm}$. The barrier is $3 \mathrm{~m}$ wide and its depth varies from 0.4 to $0.8 \mathrm{~m}$ depending on the rows of cylinders installed. The cylinders are fixed to the ground by means of a multi-layer board (plywood, plasterboard and polyester); the top fastening system, made of thin aluminum profiles, was designed to provide weak diffraction. The sound source is placed in front of the barrier at a height of $1.5 \mathrm{~m}, 1 \mathrm{~m}$ away from the closest cylinders for sound insulation measurements and $1.5 \mathrm{~m}$ away for reflection measurements [25, 26]. The microphones are arranged in a $3 \times 3$ square array (M1-M9), spaced apart $0.40 \mathrm{~m}$ both in the horizontal and vertical directions. This array is positioned parallel to the sonic crystal at a distance of $0.25 \mathrm{~m}$ either on the side of the loudspeaker (reflection measurements) or on the opposite side (sound insulation measurements). The loudspeaker always faces the central microphone of the array, M5. Since the spacing between the microphones is a multiple of the lattice constant and given the periodicity of the sample, measurements were performed in two configurations; in configuration $a$ the central microphone of the array faces the centre of a cylinder (see Fig.

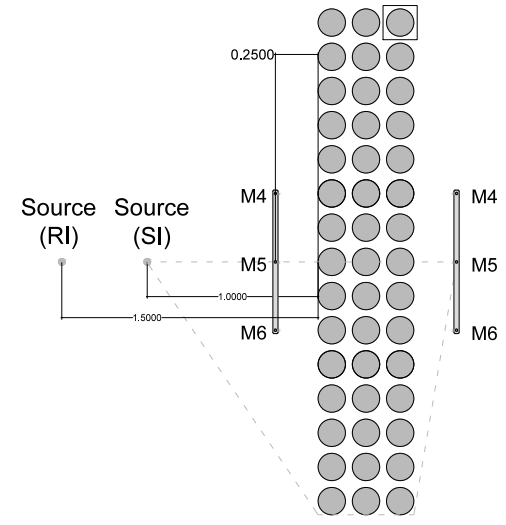

Figure 4: Experimental set-up for the barrier with 3 rows of cylinders in configuration $a$ : source and receivers positions.

4), while in configuration $b$ it faces the interstice between two adjacent cylinders. The experimental apparatus consists of (i) a 16-channel Analog to Digital converter RME M-16 AD connected to (ii) an RME Hammerfall HDSPe MADI for the A/D and D/A section, (iii) 9 Brüel \& Kjær 4935 microphones connected to (iv) a 16-channel Brüel \& Kjær 2694 preamplifier, (v) a Samson Servo 201A power amplifier and (vi) a ZIRCON loudspeaker. Since measurements were performed in a laboratory and no impulsive background noise were present, measurements were performed using ESS test signals, which were proved to be better suited in these conditions [33]. The frequency range analysed is 400$5000 \mathrm{~Hz}$.

Measurements under diffuse field conditions were performed on a $3 \times 18$ sonic crystal characterised by the same lattice characteristics tested for normal incidence (Fig. 3 b). The sonic crystal was installed in the transmission chambers of the laboratory and insulation measurements have been performed according to the standard ISO 10140-2 [34]. The source and measurement positions as well as the measurement procedure are defined by the standard ISO 10140-4 [35] standard. The measurement apparatus consists of (i) Larson\&Davis 2900 B acquisition system (ii) L\&D 2560 diffuse field 1/2" microphones (iii) L\&D PRM 900C preamplifiers (iv) $01 \mathrm{~dB}$ OMNI12 dodecahedron (v) Crown XLS1000 amplifier and (vi) noise generator. A pink noise was fed to the dodecahedron and average sound pressure level were acquired in the source and receiving room using 2 channels at the time. The reverberation time of the receiving chamber has been measured with two techniques: the interrupted noise method and the impulse 


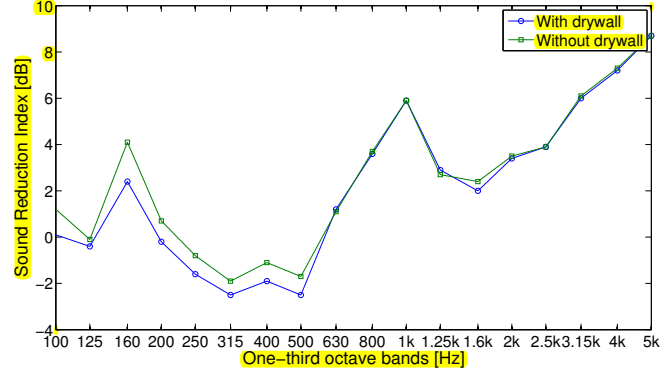

Figure 5: Sound Reduction Index measured for a 3-row sonic crystal in the transmission chamber of the University of Bologna.

response method. The two methods returned differences which are not appreciable in the evaluation of the sound reduction index and are thus not discussed in this paper. The frequency range analysed is $100-5000 \mathrm{~Hz}$.

\section{Experimental results I: the standardised criteria}

\subsection{Sound Reduction Index}

The sound reduction index can be evaluated according to the ISO 10140-2 [34] as:

$$
R=L_{1}-L_{2}+10 \log \frac{S}{A} \quad(d B)
$$

where $L_{1}(\mathrm{~dB})$ is the energy average sound pressure level measured in the source room, $L_{2}(\mathrm{~dB})$ is the energy average sound pressure level in the receiving room, $S$ is the area of the free test opening in which the test element is installed and $A$ is the equivalent absorption area in the receiving room. $A$ can be evaluated from Sabine's law by measuring the reverberation time $R T$ in the receiving room as $A=0.161 V / R T$, being $V$ the volume of the room. The EN 1793-2 allows also the calculation of a single-number rating, $D L_{R}$, used to classify noise barriers in a standardised way. This rating, which must be rounded to integer values, does not keep the frequency dependence information and assumes a fixed reference traffic noise spectrum, so that it is mainly used as a conventional reference. As derived from Eq. 1, the sound reduction index show a slight dependence on the reverberation time of the transmission chamber. Given the peculiar characteristics of the sonic crystal of being 'transparent' at certain frequencies, $R T$ measurements were performed inside the transmission chamber by (i) leaving the source and receiver chamber coupled, i.e. leaving the bare cylinders only and (ii) installing a drywall beyond the cylinders to measure the reverberation time of the receiving room as a closed volume. Figure 5 reports the sound reduction index measured using the reverberation time acquired in the two test configurations. The results show that the reverberation time measurements affect the sound reduction index only below $500 \mathrm{~Hz}$, and that the differences are smaller than $1 \mathrm{~dB}$ in almost all one-third of octave bands. As it concerns the values themselves, measurements show that the sonic crystal made of bare cylinders has poor sound insulation properties under diffuse field conditions. Qualitatively, this is in accordance with the results of Garcia-Chocano et al. [17]. A local maximum of sound attenuation is achieved at $1000 \mathrm{~Hz}$, region slightly shifted with respect to Bragg frequency, and is again followed by a local minimum. At mid-low frequencies $(250-500 \mathrm{~Hz})$ there is a region with negative values of sound reduction index. Here, since the sonic crystal is almost transparent, the measurement of its transmission loss according to this method looses its roots. However, it should be remarked that free standing noise barriers installed alongside roads or railways are exposed to a direct sound field rather than to a diffuse one, so that truly meaningful measurements should take place under a direct sound field (see next section).

\subsection{Sound Insulation index}

The sound insulation properties of the sample under a direct sound field are evaluated according to EN 17936 [26]. The sound components transmitted through the barrier and the corresponding free-field reference measurement are post-processed to compute the $S I$ index:

$$
S I_{j}=-10 \log \left\{\frac{1}{n} \sum_{k=1}^{n} \frac{\int_{\Delta f_{j}}\left|F\left[h_{t, k}(t) w_{t, k}(t)\right]\right|^{2} d f}{\int_{\Delta f_{j}}\left|F\left[h_{i, k}(t) w_{i, k}(t)\right]\right|^{2} d f}\right\}
$$

where $h_{i, k}(t)$ is the free-field impulse response at the $k$ th microphone position, $h_{t, k}(t)$ is the impulse response at the $k$-th microphone position with the barrier in between, $w_{i, k}(t)$ and $w_{t, k}(t)$ are the time windows (Adrienne temporal windows) [26] for the free-field and the transmitted components respectively at the $k$-th microphone position, $F$ denotes the Fourier transform, $j$ is the index of the $j-t$ th one-third octave frequency band, $\Delta f_{i}$ is the width of the $j-t h$ one-third octave frequency band and $n=9$ is the number of microphone positions. A more detailed description, both relative to sound insulation and sound reflection measurements, is provided in [36]. The window functions $w_{i, k}(t)$ and $w_{t, k}(t)$ are applied to the impulse responses; this permits cancellation of unwanted reflections (ground reflection amongst all) as well as diffraction from the barrier top and side edges. 


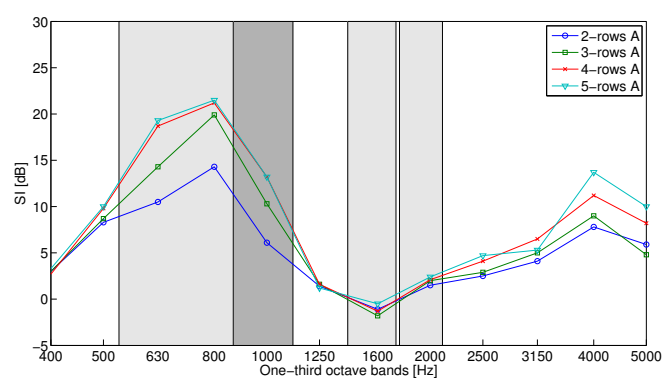

(a) SI configuration $a$

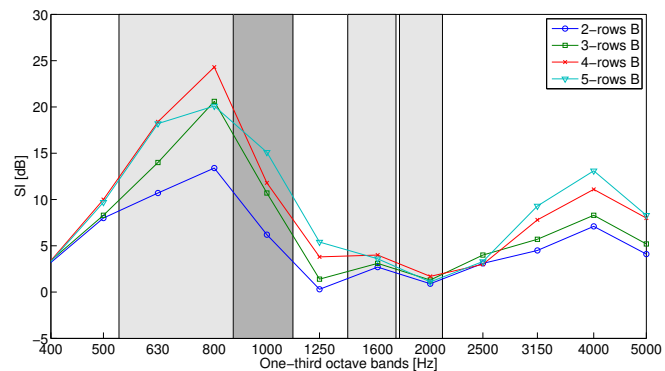

(b) $S I$ configuration $b$

Figure 6: Sound Insulation index measured in settings $a$ (the array faces the centre of the cylinders) and $b$ (the array faces the space between the cylinders). The different curves refer respectively to samples made of $2,3,4$, or 5 rows of cylinders.

According to Ref. [26] (see in particular Fig. 13), the lowest reliable frequency for a $3 \mathrm{~m}$ high barrier is about $260 \mathrm{~Hz}$. Also EN 1793-6 allows the calculation of a single-number rating, $D L_{S I}$, used to classify noise barriers in a standardised way: is has a conventional meaning like $D L_{R}$, as explained above.

Figure 6 reports the $S I$ values measured in configurations $a$ and $b$, together with the complete and partial band gaps predicted numerically, represented as dark and light grey-shaded rectangles respectively. A strong attenuation can be observed in the frequency range 500$1100 \mathrm{~Hz}$ in both configurations $a$ and $b$, i.e. with microphones facing the centre of the cylinders or the voids between cylinders. The highest $S I$ is measured in configuration $b$. At twice Bragg frequency, the $S I$ assumes values close to zero (setting $b$ ) or slightly negative (setting $a$ ). This implies the presence of a constructive interference mechanism which, given the fact that $S I$ is averaged over 9 microphone positions, cannot be due to a single-point localised anomaly. Further sound insulation measurements of sonic crystals for different measurement positions are available in [37]. The sound insulation increases with the number of rows of the sample, even though with 4 and 5 rows of cylinders the value saturates. This may be due to the limited size of the time window; while it removes the unwanted sound, it also cuts off higher order reflections from the sonic crystal elements. This poses a problem when the number of rows increases and some 'useful' signal is not captured. Indeed, for this kind of measurements the width of the time window depends on the dimensions (width and height) of the sample. For up to 3 rows of cylinders, all the significant reflections up to the $n-t h$ order are included in the time window for all rows of cylinders; for deeper samples, the $n-t h$ order reflections coming from the farther cylinders are windowed out from the impulse response and thus do not contribute to its spectral content. As a result, the $S I$ values saturate returning misleading results.

\subsection{Sound Reflection index}

The Reflection Index $(R I)$ is computed according to EN 1793-5 [25] as follows:

$$
R I_{j}=\frac{1}{n_{j}} \sum_{k=1}^{n_{j}}\left[\frac{\int_{\Delta f_{j}}\left|F\left[h_{r, k}(t) \cdot w_{r, k}(t)\right]\right|^{2} d f}{\int_{\Delta f_{j}}\left|F\left[h_{i, k}(t) \cdot w_{i, k}(t)\right]\right|^{2} d f} \cdot C_{g e o, k} \cdot C_{d i r, k}\left(\Delta f_{j}\right) \cdot C_{\text {gain }, k}\left(\Delta f_{g}\right)\right]
$$




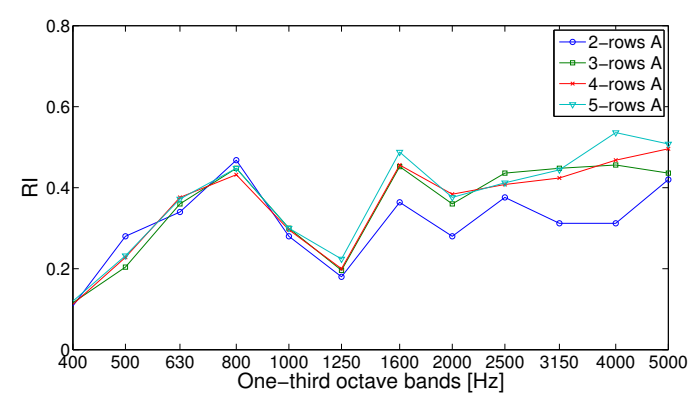

(a) RI configuration $a$

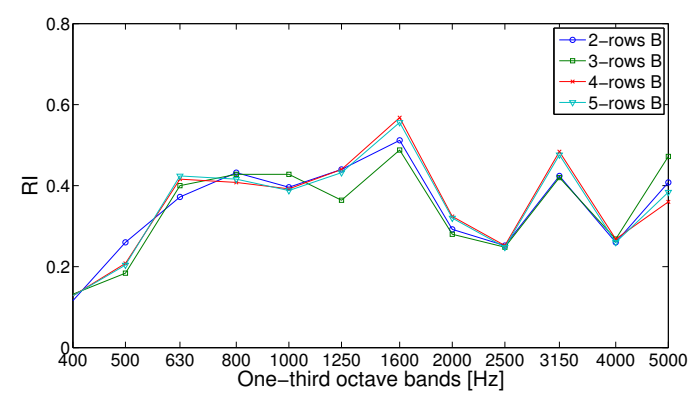

(b) RI configuration $b$

Figure 7: Reflection index measured in settings $a$ (the array faces the centre of the cylinders) and $b$ (the array faces the space between the cylinders). The different curves refer respectively to samples made of $2,3,4$, or 5 rows of cylinders.

where $h_{i, k}(t)$ is the free-field impulse response at the $k-t h$ measurement point, $h_{r, k}(t)$ is the reflected component of the impulse response at the $k-t h$ measurement point, $w_{i, k}(t)$ and $w_{r, k}(t)$ are the time windows (Adrienne temporal windows) for the free-field and the reflected components respectively, $F$ is the symbol of the Fourier transform, $j$ is the index of the $\mathrm{j}$-th one-third octave frequency band (between $100 \mathrm{~Hz}$ and $5000 \mathrm{~Hz}$ ), $\Delta f_{i}$ is the width of the $\mathrm{j}$-th one-third octave frequency band, $n$ is the number of microphone positions on which to average. With reference to the $k-t h$ measurement point, $C_{g e o, k}$ is the correction factor for the geometrical divergence, $C_{d i r, k}\left(\Delta f_{j}\right)$ is the correction factor for sound source directivity, $C_{\text {gain }, k}\left(\Delta f_{g}\right)$ is the correction factor relative to the loudspeaker amplification and the microphones sensitivity and $\Delta f_{g}$ is the frequency range encompassing the one-third octave frequency bands between 500 and $2000 \mathrm{~Hz}$.

Figure 7 shows the $R I$ measured in configurations $a$ and $b$. There is a remarkable difference between the $R I$ evaluated in the two configurations, especially concerning the general trend. Configuration $a$ displays a local maximum in the one-third of octave band centred at $800 \mathrm{~Hz}$, low values at $1250 \mathrm{~Hz}$ and increase at higher frequencies. In configuration $b$ the $R I$ displays a regular trend in the Bragg frequency range, settling to 1 . While $S I$ values do not change dramatically shifting between configurations $a$ and $b, R I$ measurements are strongly affected by the position of the microphone relative to the sample. While the impulse response in transmission is representative of a multiple scattering process, the impulse response measured in reflection is strongly affected by the first reflections. The energy related to the very first reflections determines significantly the spectral content of the signal, while subsequent reflections have smaller relative weight. This might explain both the dependence on the position and the independence from the number of rows. It is interesting to note that the spacing between the microphones is a multiple of the lattice constant, i.e. when the central microphone of the array faces the centre of a cylinder, all microphones face the centre of the cylinders, under different incidence angles: this might have amplified this effect. The Reflection Index seems not to be closely dependent on the number of rows of the sonic crystal. Also this issue might be related to the windowing procedure. For the Reflection Index, the application of the time window is even more critical than in the calculation of SI; here the time window is centred on the arrival of the direct sound, thus multiple scattering components coming from the farthest cylinders might be easily windowed out.

\section{Experimental results II: single point measure- ments and FE predictions}

\subsection{Single point measurements}

In order to investigate the local behaviour of the sonic crystal, a single-point sound insulation index is computed for microphone M5, located in the centre of the array. A modified Adrienne window is applied to the impulse responses according to the specifications reported in EN 1793-5 [25] and EN 1793-6 [26] and the single point $S I$ and $R I$ are evaluated as the ratio of the power spectra computed in narrowband, without considering any correction factor. The results are shown in Figures 8 and 9. The insulation properties of the sample are not strongly affected by the position of the microphone relative to the cylinders around the Bragg frequency, while at higher frequency distinctions arise between configurations $a$ and $b$. In particular, the region of null or slightly negative $S I$ values is wider in configuration $a$. It is worth noting that the barrier with only two rows of cylinders already generates an appreciable mechanism of wave interference responsible for the band gap. The 


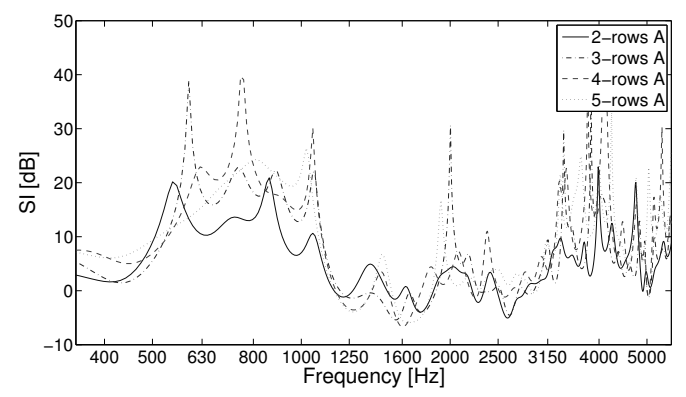

(a) SI configuration $a$

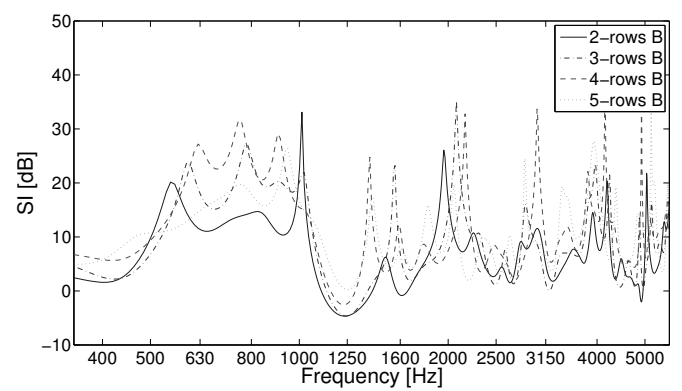

(b) $S I$ configuration $b$

Figure 8: Single point $S$ I measured at microphone M5 in configurations $a$ and $b$. The different curves refer respectively to samples made of $2,3,4$, or 5 rows of cylinders.

reflection index oscillates in frequency around the value of 1 and shows to be strongly dependent on the position of the microphone. It reaches a local maximum of 1.7 at around $700 \mathrm{~Hz}$, significantly below Bragg frequency.

\subsection{FE predictions accuracy}

The band structure analysis shows that filtering properties exist in an infinite sonic crystal barrier made of hollow PVC cylinders with a proper spatial arrangement. However, in order to quantify the magnitude of such attenuation, the finite length of the sonic crystal must be taken into account. To this end, FE timeharmonic analyses are performed on a finite-size sonic crystal. The sound source (S), the evaluation point (M5) and their relative positions with respect to the barrier under test are set in accordance to EN 1793-6 [26] and EN 1793-5 [25]. Perfectly-Matched Layers (PML) with a thickness of $1 \mathrm{~m}$ are symmetrically set around the computational domain in order to avoid sound reflections from the boundaries. Due to the similarity in the band structures of Figs. 2a and 2b, in these simulations the hollow PVC cylinders are modeled as filled to ease the generation of the FEM mesh. Neumann boundary conditions (acoustically hard surfaces) are applied to their

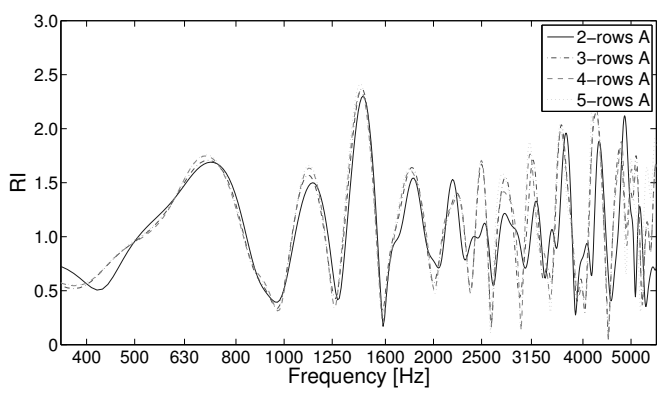

(a) RI configuration $a$

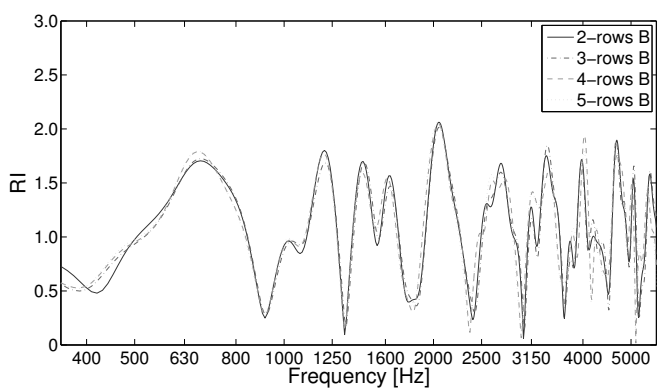

(b) $R I$ configuration $b$

Figure 9: Single point $R I$ measured at microphone M5 in configurations $a$ and $b$. The different curves refer respectively to samples made of $2,3,4$, or 5 rows of cylinders.

perimeter. In order to provide accurate results up to $2500 \mathrm{~Hz}$, a mesh made of constant strain triangular elements of maximum edge size of $0.01 \mathrm{~m}$ is set. Sound attenuation, evaluated as the difference between the spectra with and without the barrier, is extracted at point M5 in the two testing configurations $a$ and $b$.

The match between FE predictions and single point measurements is presented in Fig. 10. The single point $S I$ shows a good agreement with the numerical calculations, locating accurately the band gap frequency ranges. In particular, configuration $b$ shows the best fit. The constructive interference that was detected in the $S I$ evaluation (Fig. 6) and here, is also clearly predicted by FE calculations in the single point measurements. The behaviour of the sample in reflection is accurately predicted at Bragg frequency, while differences increase shifting towards higher frequencies.

The comparison between numerical predictions and experimental measurements is made consistent by a combination of assumptions adopted in the FE models and in the experimental acquisition procedure. In particular: (i) the single point $S I$ is computed at point M5, that lies at the same height of the sound source; (ii) the frequency steps in the time harmonic analyses 


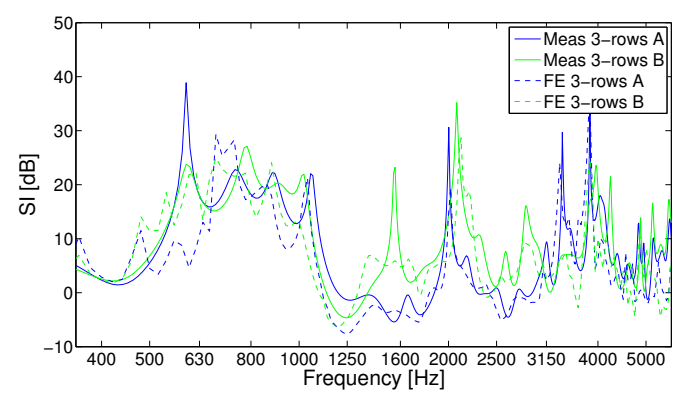

(a) Single point $S I$

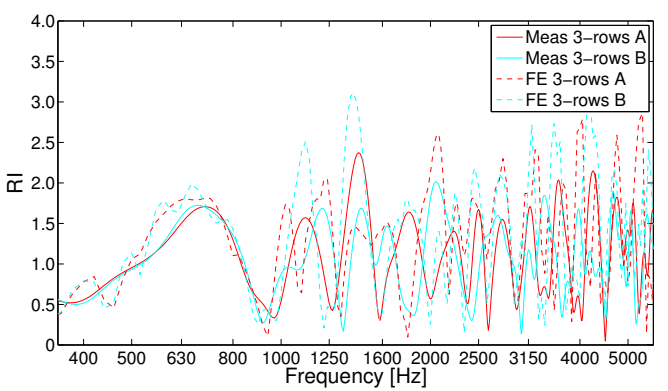

(b) Single point $R I$

Figure 10: FE-computed sound attenuation (a) and sound reflection (b) at microphone M5 for a 3-rows sonic crystal in configurations $a$ and $b$.

are set in accordance to the maximum frequency resolution achievable due to the aforementioned windowing procedure over the impulse responses; (iii) the experimental windowing procedure is mimicked in the numerical model introducing PMLs (which neglects the reflections from the boundaries), limiting the domain in width (thus preventing edge diffraction effects) and assuming a 2D plane strain domain (to avoid ground and top barrier edge reflections). The sound source used to perform experimental measurements behaves as a point source; this explains the good agreement which was found between measurements and FE simulations performed with a point source. While band structures and FE calculations performed on finite size samples with a plane wave source show an attenuation at twice Bragg frequency [40], measurements and predictions involving a point source do not display a Bragg band gap at this frequency but a pass band occurs instead.

\subsection{Top diffraction effect}

Another interesting feature of the sound insulation is the effect of the diffraction from the top of this periodic structure: can it be considered negligible? A further set of measurements was conducted on a sonic crystal by

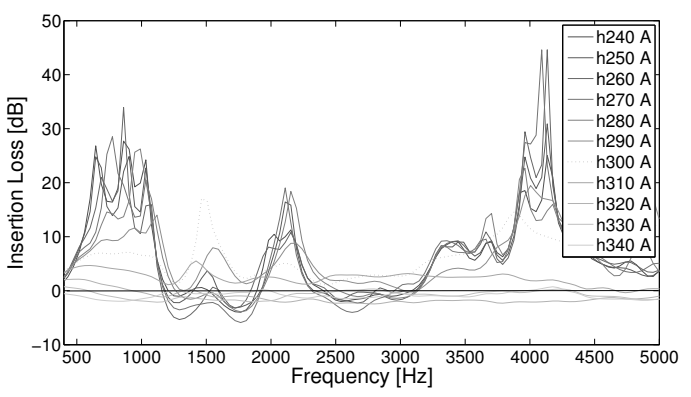

(a) Configuration $a$

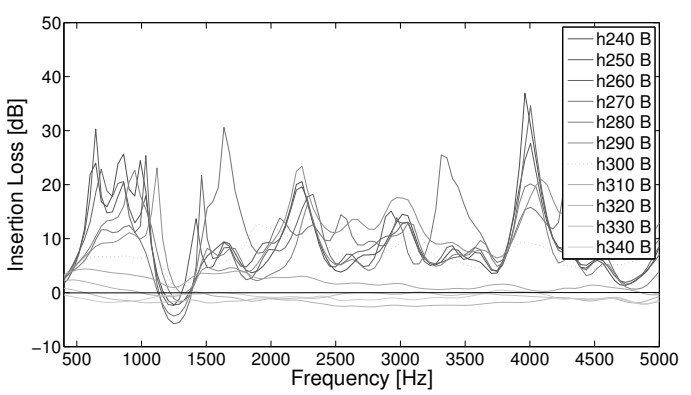

(b) Configuration $b$

Figure 11: Insertion Loss measured at different heights from the barrier. The insertion loss measured at a height of $3 \mathrm{~m}$, i.e. the height of the barrier, is represented as a thicker dashed line while the IL measured at other heights are reported in grey scale starting from the lower (black) to the highest measurement point (light grey). The mic height relative to each curve is reported in the legend (h 240 for instance).

shifting the source and the receiver upwards and leaving the time window unchanged. This excluded ground reflections and side diffraction and to compute the combined effects of sound transmission and top diffraction. The insertion loss is presented in Fig. 11 in configurations $a$ and $b$. A huge difference is found for top diffraction between the two configurations. When the microphone faces the cylinder (config. $a$ ), the shift of the microphone causes a drop in $S I$ in the Bragg frequency and a peak around $2000 \mathrm{~Hz}$. A component emerges at $1600 \mathrm{~Hz}$, the region in which the $S I$ displays negative values. Higher frequency $S I$ values are less affected by the shift, except for the heights at which the source and the microphone are sensitively higher than the barrier. In configuration $b$ the drop around Bragg frequency occurs as well, and the dip at $1250 \mathrm{~Hz}$ is identified equally from all measurements, irrespective of the diffraction effect. The behaviour at higher frequency is also more homogeneous than in case $a$, except again for the last configurations. 


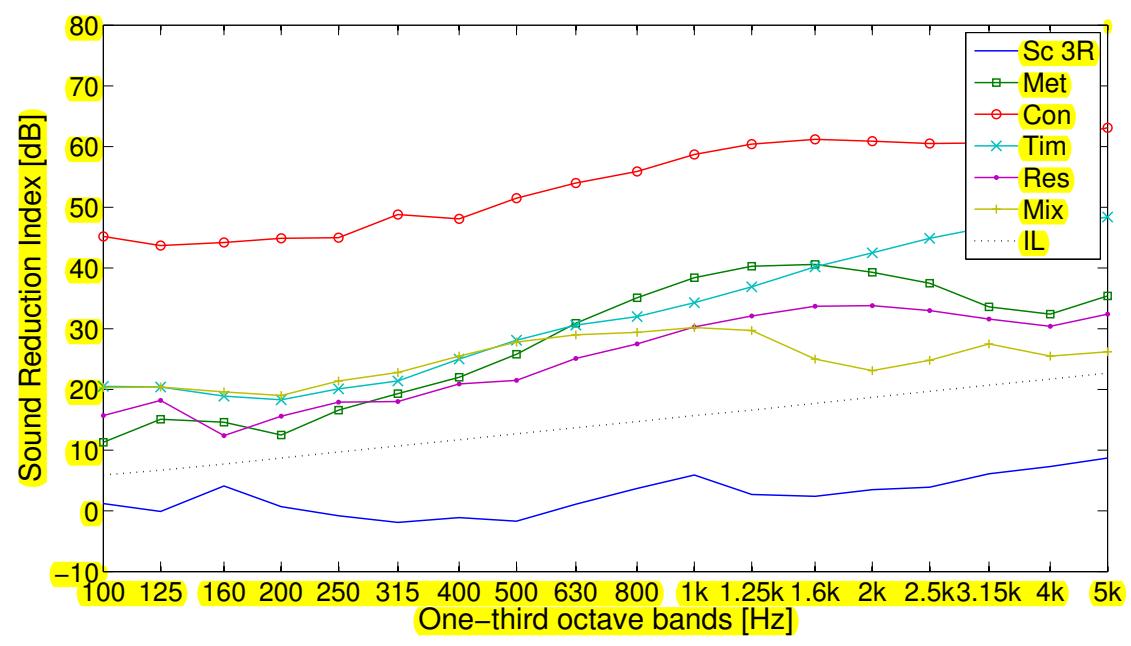

Figure 12: Comparison between the sound reduction index measured in coupled laboratory reverberation rooms (EN 1793-2) for a 3-rows sonic crystal (SC 3R) and standard noise barriers: metallic barrier (MET), concrete barrier (CON), timber barrier (TIM), plastic resin barrier (RES), mixed metallic and acrylic barrier (MIX). The dashed line (IL) is the insertion loss due to the top edge diffraction over a noise barrier $3 \mathrm{~m}$ high calculated according to [39]. The values of $D L_{R}$ are reported in brackets.

\section{Discussion}

The standards EN 1793-5 and EN 1793-6 determine the measurement procedure to characterise sound reflection and sound insulation of acoustic screens for normal incidence excluding any diffraction component. Considering the lattice under study, the Bragg scattering is expected to occur around $860 \mathrm{~Hz}$. This is the region in which the insulation and reflection indices should display a local maximum, in the first case due to the interference occurring between incident and backscattered waves inside the crystal and in the second case to the constructive interference occurring between incident and scattered waves with a phase shift comparable to the lattice constant.

The $S I$ values identify Bragg scattering clearly, locating accurately the stop band as predicted from the band structure. The application of the time window on one side allows to neglect ground reflection and edge diffraction; on the other hand it limits the possibility to investigate these structures when the number of rows increases. With the lattice constant of $0.2 \mathrm{~m}$, this significance threshold was identified in 3 rows of cylinders; after this number, an increase in depth did not correspond to an increase in insulation. While Bragg band gap is well identified, at twice Bragg frequency no insulation properties are found as predicted by the band structures and by FE computation. Instead, a null or negative $S I$ settles, that is related to a constructive interference which emerges even if averaged over the 9 microphone positions. This fact should be further investigated. It may be due to the fact that at this frequency, where the wavelength is equal to the lattice constant, the gap between cylinders acts as a sort of wave guide producing some amplification.

Reflection index shows to have a strong dependence on the position of the microphone. The values of the $R I$ averaged over 9 microphones and the single point $R I$ show that, apart from correction factors, the oscillating behaviour is depending totally on the position of the microphone; in fact it settles to regular trends when averaged. The reflection coefficients were found to be also quite independent of the number of rows of the crystal. This might be explained considering that in reflection measurements the direct sound is a key component in the spectral content of the impulse response, as well as the early reflections; subsequent $n$ - th order reflections play a minor role. The application of the time window for reflection impulse response is also more critical, being the direct sound involved, as all useful multiple scattering components are shifted forward in time.

The analysis on the top diffraction effect provided by sonic crystals was meant to understand the effectiveness of the application of the time window in the determination of the insulation properties of a sonic crystal. By using the same time window and shifting the source and the microphone upwards, the intent was to exclude the side diffraction and ground reflection and to consider increasingly the top diffraction effect. As a general trend, by increasing the height the insulation 
properties dropped quite linearly. When the source and the receiver were set at the same height of the cylinders, the sound attenuation at grazing incidence is about $7 \mathrm{~dB}$ at Bragg frequency, greater than the $5 \mathrm{~dB}$ predicted by Kurze and Anderson [39], which might suggest an interesting effectiveness of sonic crystals used as low-height noise barriers.

The main strength of using standardised indices is that they allow comparisons between different samples. In Figures 12 and 13 the performance of a sonic crystal noise barrier is tested versus common noise barriers (some of them were already presented in Ref. [27]).

Fig. 12 reports the sound reduction index values for lightweight and heavyweight noise barriers existing in the market together with the values measured for a 3rows sonic crystal. In a diffuse sound field the performance of the sonic crystal made of rigid cylinders are not comparable with the usual noise barriers. Anyway, it should be considered that a diffuse sound field is not totally representative of the real in situ performance of a noise barrier, as previously discussed. The poor insulation provided under these test conditions agrees with the results reported in a previous work [17]. In that work, the calculation of the transmission loss generated by rigid cylinders shows a flat trend up to $300 \mathrm{~Hz}$, an increase in insulation that reaches its maximum at $600 \mathrm{~Hz}$ and settles around $5 \mathrm{~dB}$ for increasing frequency, returning a $D L_{R}=4 \mathrm{~dB}$. The frequency trend highlighted in the present work differs significantly; as shown in Fig. 5 the high frequency attenuation is very low and sound insulation at Bragg frequency is more evident, though shifted towards higher frequencies. Moreover, the $D L_{R}$ measured for this array is $2 \mathrm{~dB}$. This might be due to the different modulation in lattice constant alongside the depth of the sample to which Ref. [17] refers. Improvements of the sound insulation performance under diffuse conditions can be achieved by adding absorptive material, i.e. making sound absorption in a reverberant space the dominating attenuation mechanism.

Fig. 13 reports the $S I$ values for lightweight and heavyweight noise barriers existing in the market together with the values measured for a 3-rows sonic crystal. The barriers are the same tested under diffuse field conditions reported in Fig. 12 but now the free-field method allows separate measurements across the acoustic elements (panels) and across the posts. Data according to EN 1793-6 are available on the full frequency range $100 \mathrm{~Hz}-5000 \mathrm{~Hz}$ but as far as it concerns the sonic crystal, only data measured in the range $400 \mathrm{~Hz}$ $5000 \mathrm{~Hz}$ should be considered to account for the modified Adrienne time window used to characterise the sample. The measurements across the post for the plas- tic resin barrier (RES), the metallic barrier (MET) and the mixed metal/acrylic barrier (MIX) display sound insulation values which are of the same order of magnitude of the maximum $S I$ measured for a sonic crystal, while the heavy metal and concrete elements show higher sound insulation values. For the mixed barrier, the $S I$ values measured across the posts (MIX P) drop dramatically $(9 \mathrm{~dB})$ in comparison to the values across the elements (MIX E); for the concrete barrier the drop is $19 \mathrm{~dB}$ [27]. This suggests that the actual sound insulation behind a real road barrier may be severely limited by the sound leakage at the panel-post junction. More in general, the sound insulation of the marketed noise barriers seems to exceed the real needs. In order to highlight the point, the black dotted line in Fig. 13 shows the theoretical insertion loss achievable for a given geometry of the barrier due to the top diffraction according to Ref. [39]. Following the analytic expression proposed in that work, the insertion loss has been calculated considering a point source placed at a height of $1.5 \mathrm{~m}$ and at a distance of $3 \mathrm{~m}$ from the barrier, while the receiver is a point located at a height of $1.5 \mathrm{~m}$ (approximately a window at the ground floor) and at a distance of $10 \mathrm{~m}$ from the barrier. This theoretical value of insertion loss shows to be interesting for pointing out the effectiveness of sonic crystal noise barriers. The overall effect of any noise barrier is dominated by the sound diffracted over the top edge and the results show that in fact, all other kinds of barrier are oversized with respect to their insulation properties. With a proper enhancement of the sound insulation properties of such crystals, achievable by all methods reported in the above literature review, sonic crystals might turn out to be an interesting substitute for common noise barriers.

\section{Conclusions}

In this work, the existence of band gaps in sonic crystal noise barriers has been numerically investigated and experimentally validated for multiple configurations. Sound insulation and reflection properties have been performed according to the European standards for in situ measurements on road traffic noise barriers for normal incidence, EN 1793-5 and EN 1793-6 and for laboratory measurements under diffuse field conditions, EN 1793-2.

The measurements performed for free-field conditions show sound insulation values up to $24 \mathrm{~dB}$ in the target frequency range and reflection values around 1 , with a strong dependence on the measurement position. The processing procedure that characterised free field 


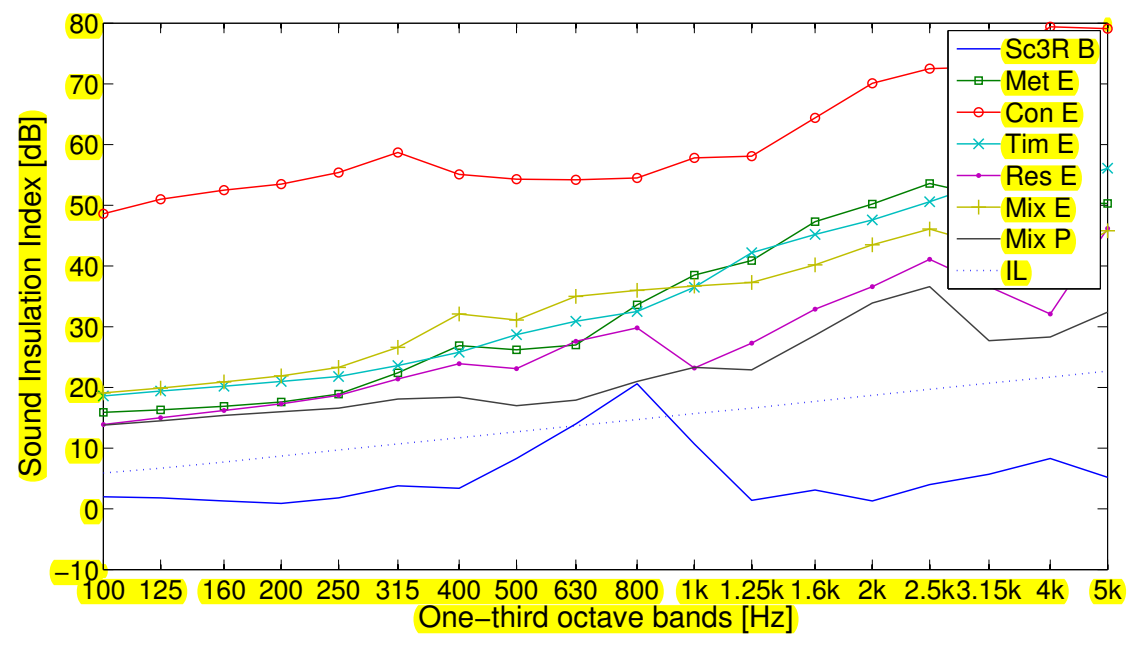

Figure 13: Comparison between the Sound insulation index measured in free-field (EN 1793-6) for a 3-rows sonic crystal (SC 3R) and standard noise barriers: metallic barrier (MET), concrete barrier (CON), timber barrier (TIM), plastic resin barrier (RES), mixed metallic and acrylic barrier (MIX). The letter E or P after the barrier label denotes a measurement across the acoustic elements or a post, respectively. The dashed line (IL) is the insertion loss due to the top edge diffraction over a noise barrier $3 \mathrm{~m}$ high, calculated according to [39]. The values of $D L_{S I}$ are reported in brackets.

measurements allows to compute only the sound components transmitted through the sample by windowing out the ground reflection and the edge diffraction, leading to an intrinsic characterisation of the screen. On the other side, when evaluating the sound insulation properties of sonic crystals composed of increasing number of rows, what was considered an advantage becomes a constraint. In fact, the finite size of the time window excludes higher order reflections and early reflections coming from the farthest cylinders. This translates into a saturation of the $S I$ value after 3 rows of cylinders for the lattice under study and on a poor dependence of the $R I$ on the number of rows.

The stop-band properties of sonic crystals have been further investigated locally. Sound attenuation and reflection have been computed on a single measurement point and the results were compared with the attenuation predicted by a finite-size FE model. Experimental results are in very good agreement with the numerical ones, locating accurately the band gap frequency range and capturing the sharpening of the transmission power spectrum as the considered number of cylinder rows increases.

The availability of standardised values allowed a direct comparison of the sound insulation and reflection properties of the sonic crystal noise barriers related to other classical screening techniques. This comparison was held considering metal, concrete, timber, plastic resin and mixed metal/acrylic "common" noise barri- ers compared to sonic crystal noise barriers. The results showed a good performance of the sonic crystal in the designed stop band, especially considering the comparison between the $S I$ values and the maximum achievable values of insertion loss due to the top diffraction. The measurements performed under diffuse field conditions identify poor insulation properties of sonic crystals compared to other noise barriers, but since barriers are mounted in free field, the characterisation under diffuse field conditions is less crucial.

\section{Acknowledgements}

This research project was supported by CIRI-MAM (Advanced Mechanics and Materials) and CIRI-EC (Building and Construction), POR-FESR 2007-2013. M.M. has received funding from the European Union Horizon 2020 research and innovation programme under the Marie Sklodowska-Curie grant agreement N. 658483. The authors gratefully acknowledge the support of Eng. P. Guidorzi for the measurement session, Eng. S. De Cesaris for the data processing and Mr. G. Bartolotta for the installation of the full-scale samples.

\section{References}

[1] R. Màrtinez-Sala, J. Sancho, J. V. Sànchez, V. Gomez, J. Llinares and F. Meseguer. Sound attenuation by sculpture. Nature 378 (6554), 241, 1995. 
[2] J. V. Sànchez-Pèrez, D. Caballero, R. Màrtinez-Sala, C. Rubio, J. Sànchez-Dehesa, F. Meseguer, J. Llinares and F. Gàlvez. Sound attenuation by a two-dimensional array of rigid cylinders. Phys. Rev. Lett. 80, 5325-5328, 1998.

[3] M. S. Kushwaha and B. Djafari-Rouhani. Sonic stop-bands for periodic arrays of metallic rods: honeycomb structure. J. Sound Vib. 218 (4), 697-709, 1998.

[4] Y. Y. Chen and Z. Ye. Acoustic attenuation by two-dimensional arrays of rigid cylinders. Phys. Rev. Lett. 87 (18), 184301, 2001.

[5] L. Sanchis, F. Cervera, J. Sànchez-Dehesa, J. V. Sànchez-Pèrez, C. Rubio and R. Martìnez-Sala. Reflectance properties of twodimensional sonic band-gap crystals. J. Acoust. Soc. Am. 109 (6), 2598-2605, 2001.

[6] J. O. Vasseur, P. A. Deymier, A. Khelif, Ph. Lambin, B. DjafariRouhani, A. Akjouj, L. Dobrzynski, N. Fettouhi and J. Zemmouri. Phononic crystal with low filling fraction and absolute acoustic band gap in the audible frequency range: A theoretical and experimental study. Phys. Rev. E 65, 056608, 2002.

[7] H. Pichard, O. Richoux and J. P. Groby. Experimental demonstrations in audible frequency range of band gap tunability and negative refraction in two-dimensional sonic crystal. J. Acoust. Soc. Am. 132 (4), 2816-2822, 2012

[8] S. Castineira-Ibànez, C Rubio, V. Romero-Garcìa, J. V. Sànchez-Pèrez, and L. M. Garcìa-Raffi. Design, manufacture and characterization of an acoustic barrier made of multiphenomena cylindrical scatterers arranged in a fractal-based geometry. Archives of Acoustics 37 (4), 455-462, 2012.

[9] V. Romero-Garcìa, C. Lagarrigue, J.-P. Groby, O. Richoux and V. Tournat. Tunable acoustic waveguides in periodic arrays made of rigid square-rod scatterers: theory and experimental realization. J. Phys. D: Appl. Phys. 46 (30), 305108, 2013.

[10] R. Martìnez-Sala, C. Rubio, L. M. Garcìa-Raffi, J. V. SànchezPèrez, E. A. Sànchez-Pèrez, J. Llinares. Control of noise by trees arranged like sonic crystals. J. Sound Vib. 291, 100-106, 2006.

[11] J. V. Sànchez-Pèrez, C. Rubio, R. Màrtinez-Sala, R. SànchezGrandia and V. Gomez. Acoustic barriers based on periodic arrays of scatterers. Appl. Phys. Lett. 81 (27), 5240-5242, 2002.

[12] X. Hu, C. T. Chan and J. Zi. Two-dimensional sonic crystal with Helmholtz resonators. Phys. Rev. E 71, 055601, 2005.

[13] A. Krynkin, O. Umnova, A. Chong, S. Taherzadeh and K. Attenborough. Scattering by coupled resonating elements in air. J. Phys. D: Appl. Phys. 44, 125501, 2011.

[14] D. P. Elford, L. Chalmers, F. V. Kusmartsev and G. M. Swallowe. Matryoshka locally resonant sonic crystal. J. Acoust. Soc. Am. 130 (5), 2746-2755, 2011.

[15] C. Lagarrigue, J. P. Groby and V. Tournat. Sustainable sonic crystal made of resonating bamboo rods. J. Acoust. Soc. Am. 133 (1), 247-254, 2013.

[16] V. Romero-Garcìa, A. Krynkin, L. M. Garcia-Raffi, O. Umnova, and J. V. Sànchez-Pèrez. Multi-resonant scatterers in sonic crystals: Locally multi-resonant acoustic metamaterials. J. Sound Vib. 332 (1), 184-198, 2013.

[17] V. M. Garcìa-Chocano, J. Sànchez-Dehesa. Optimum control of broadband noise by arrays of cylindrical units made of a recycled materials. Appl. Acoust. 74, 58-62, 2013.

[18] V. M. Garcìa-Chocano, S. Cabrera, J. Sànchez-Dehesa. Broadband sound absorption by lattices of microperforated cylindrical shells. Appl. Phys. Lett. 101, 184101, 2012.

[19] J. Sànchez-Dehesa, V. M. Garcìa-Chocano, D. Torrent, F. Cervera, S. Cabrera, F. Simon. Noise control by sonic crystals barriers made of recycled materials. J. Acoust. Soc. Am. 129, 1173-1183, 2011.

[20] O. Umnova, K. Attenborough and C. M. Linton. Effects of porous covering on sound attenuation by periodic arrays of cylinders. J. Acoust. Soc. Am. 119 (1), 278-284, 2006.
[21] V. Romero-Garcìa, J. V. Sànchez-Pèrez, L. M. Garcìa-Raffi, J. M. Herrero, S. Garcìa-Nieto and X. Blasco. Hole distribution in phononic crystals: Design and optimization. J. Acoust. Soc. Am. 125 (6), 3774-3783, 2009.

[22] L.Y. Wu and L. W. Chen. Wave propagation in a 2D sonic crystal with a Helmholtz resonant defect. J. Phys. D: Appl. Phys. 43, 055401, 2010.

[23] EN 14388. Road traffic noise reducing devices. Specification. CEN, Brussels, 2015

[24] EN 1793-2. Road traffic noise reducing devices - Test method for determining the acoustic performance - Part 5: Intrinsic characteristics of airborne sound insualtion under diffuse sound field conditions, CEN, Brussels, 2012.

[25] EN 1793-5. Road traffic noise reducing devices - Test method for determining the acoustic performance - Part 5: Intrinsic characteristics - In situ values of sound reflection under direct sound field conditions, CEN, Brussels, 2014.

[26] EN 1793-6. Road traffic noise reducing devices - Test method for determining the acoustic performance - Part 6: Intrinsic characteristics - In situ values of airborne sound insulation under direct sound field conditions, CEN, Brussels, 2012.

[27] M. Garai and P. Guidorzi. European methodology for testing the airborne sound insulation characteristics of noise barriers in situ: Experimental verification and comparison with laboratory data. J. Acoust. Soc. Am. 108 (3), 1054-1067, 2000.

[28] U. Sandberg. The multi-coincidence peak around $1000 \mathrm{~Hz}$ in tyre/road noise spectra. Proc. 5th European Conference on Noise Control, 2003.

[29] Comsol Multiphysics - release 4.3a, www.comsol.com, 2012.

[30] M. Miniaci. Behaviour and applications of elastic waves in structures and metamaterials. PhD thesis - Alma Mater Studiorum, University of Bologna, Bologna, 2014.

[31] M. Miniaci, A. Marzani, N. Testoni and L. De Marchi. Complete band gaps in a polyvinyl chloride (PVC) phononic plate with cross-like holes: numerical design and experimental verification. Ultrasonics 56, 251-259, 2015.

[32] Y. Pennec, J. O. Vasseur, B. Djafari-Rouhani, L. Dobrzyǹski and P. A. Deymier. Two-dimensional phononic crystals: Examples and applications. Surf. Sci. Rep. 65 (8), 229-291, 2010.

[33] M. Garai, P. Guidorzi. Sound reflection measurements on noise barriers in critical conditions, Building and Environment 94 (2), 752-763, 2015

[34] EN ISO 10140-2. Acoustics - Laboratory measurement of sound insulation of building elements - Part 2: Measurement of airborne sound insulation, ISO, Geneva, 2010.

[35] EN ISO 10140-2. Acoustics - Laboratory measurement of sound insulation of building elements - Part 4: Measurement procedures and requirements, ISO, Geneva, 2010.

[36] P. Guidorzi and M. Garai. Advancements in sound reflection and airborne sound insulation measurement on noise barriers. O. J. A. 3, 25-38, 2013.

[37] F. Morandi, P. Guidorzi, M. Miniaci, A. Marzani and M. Garai. Acoustic measurements on sonic crystal barriers. Energy Procedia 78, 134-139, 2015.

[38] M. Garai, E. Schoen, G. Behler, B. Bragado, M. Chudalla, M. Conter, J. Defrance, P. Demizieux, C. Glorieux and P. Guidorzi. Repeatability and reproducibility of measurements of sound reflection and airborne sound insulation index of noise barriers. Acta Acustica united with Acustica 100 (6), 1186-1201, 2014.

[39] U. J. Kurze, G. S. Anderson. Sound attenuation by barriers. Appl. Acoust. 4, 35-53, 1971.

[40] F. Morandi, S. De Cesaris, M. Garai, M. Miniaci, A. Marzani. Experimental evidence of band gaps in periodic structures. Proc. of Euronoise 2015, Maastricht, 2015. 